\section{Stereotactic radiosurgery alone for limited brain metastases: are we ready for prime time?}

\author{
"Ultimately, the historical approach of brain metastases management \\ based strictly on a number of lesions is overly simplistic."
}

\section{Simon S Lo ${ }^{*, 1}$, Kristin J Redmond ${ }^{2}$, Eric L Chang ${ }^{3}$, Matthew Foote ${ }^{4}$, Jonathan PS Knisely ${ }^{5} \&$ Arjun Sahgal ${ }^{6}$}

First draft submitted: 18 September 2015; Accepted : 22 September 2015; Online: 18 December 2015

Whole brain radiotherapy (WBRT) has traditionally been the standard treatment for brain metastases [1]. More recently, patients with limited brain metastases are being offered stereotactic radiosurgery (SRS), with or without WBRT, in an attempt to improve survival and functional outcomes. A recent editorial has suggested that the sun is setting on WBRT and SRS is rising to be the standard of care [2]. This editorial summarizes the data from individual international randomized trials and a meta-analysis regarding the role of SRS in patients with limited brain metastases, and will focus on its role as a definitive therapy.

For patients with limited brain metastases, definitive local therapy in addition to WBRT has been demonstrated to improve local control and overall survival compared with WBRT alone [3-5]. In the Radiation Therapy Oncology Group (RTOG) 9508 trial, patients with 1-3 new brain metastases were randomized to WBRT alone or WBRT plus SRS. A survival advantage was observed in patients with a single brain metastasis who were treated with WBRT plus SRS [5]. Furthermore, the addition of SRS resulted in significant improvements in local tumor control, Karnofsky Performance Status (KPS) and reducing steroid dependency and did not increase acute or late toxicities [5].

Reinforcement of the importance of local control for brain metastasis patients was provided by two Phase III trials comparing WBRT with or without surgical resection in patients with solitary metastases [3,4]. In these studies, both local control and survival were improved by more aggressive local therapy. Interestingly and importantly, a trial comparing resection with or without WBRT showed no survival advantage to the addition of WBRT, even though decreased distant brain failures were observed [6]. This result likely reflects both the efficacy of SRS and WBRT as salvage therapies and the competing risk of extracranial disease

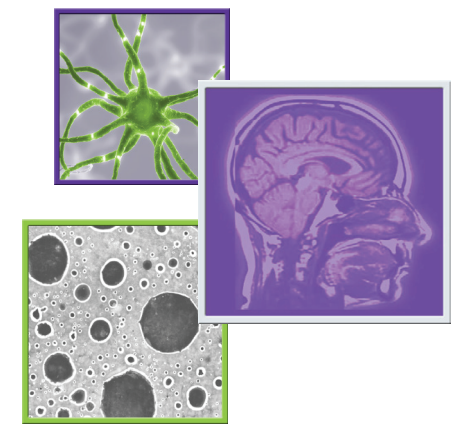

\section{KEYWORDS \\ - limited brain metastases \\ - stereotactic radio surgery \\ - whole-brain radiotherapy}

“...stereotactic radiosurgery alone results in not only better neurocognitive function but also quality of life."

'Department of Radiation Oncology, University Hospitals Seidman Cancer Center, Case Comprehensive Cancer Center, Cleveland, OH, USA

2Department of Radiation Oncology \& Molecular Radiation Sciences, Johns Hopkins University, Baltimore, MD, USA

${ }^{3}$ Department of Radiation Oncology, Norris Cancer Center \& Keck School of Medicine at University of Southern California, Los Angeles, CA, USA

${ }^{4}$ Department of Radiation Oncology, Princess Alexandra Hospital, School of Medicine, University of Queensland, Queensland, Australia

${ }^{5}$ Department of Radiation Medicine, North Shore - Long Island Jewish Health System, Hofstra University School of Medicine, Lake Success, NY, USA

${ }^{6}$ Department of Radiation Oncology, Sunnybrook Health Sciences Centre, University of Toronto, Toronto, ON, Canada *Author for correspondence: Tel.: +1 1216286 6740; simon.lo@uhhospitals.org

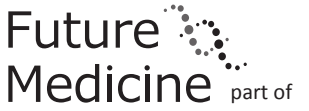
isg 
"Overall, when all patients were analyzed, no survival benefit to WBRT was observed despite lower rates of local and distant brain failure." progression on survival. Nonetheless, they demonstrate the importance of local control in the CNS in patients with limited brain metastases.

Astute investigators then explored the possibility of withholding WBRT in this group of favorable patients (1-4 metastases, KPS $\geq 70$ and controlled extracranial disease), and treating with SRS alone. There have now been four randomized Phase III trials comparing WBRT + SRS to SRS alone for patients with 1-3 or 1-4 brain metastases [7-10]. The first reported was the Japanese Phase III trial for patients with $1-4$ brain metastases (JROSG 99-1). This trial showed that omission of WBRT results in worse intracranial disease control, without negatively impacting overall survival [7]. Of note, the trial was not powered for survival and the primary end point was intracranial relapse. More patients received salvage treatment in the SRS alone group, but neurological death rates were similar between arms [7]. Neurocognitive function was prospectively evaluated with serial Mini-Mental State Examination (MMSE), and no difference in MMSE scores were observed between the two arms [7]. A secondary analysis suggested that relapse may yield deterioration in MMSE scores and this was presented as an argument to favor WBRT + SRS [11]. However, these data also revealed that in patients treated with SRS alone, deterioration in MMSE scores from intracranial relapses returned to baseline with salvage therapy. By contrast, treatmentinduced deterioration in MMSE score following WBRT plus SRS were refractory to medical and other interventions. Ultimately, however, the conclusions regarding neurocognitive impact drawn from this study were limited since the MMSE is an insensitive test for neurocognition.

The next Phase III trial to be reported came from the MD Anderson Cancer Center (MDACC) and also compared SRS alone to WBRT plus SRS. In this trial, the primary end point was performance on the Hopkins Verbal Learning Test - revised (HVLT-R), a validated measure of short-term verbal memory. This trial was stopped early after an interim analysis showed that patients treated with SRS alone had a significantly greater probability of retaining memory function at 4 months post-treatment, as compared with those treated with WBRT plus SRS [8]. No detrimental effect on overall survival was observed with the omission of WBRT for patients with 1-3 brain metastases despite a greater rate of intracranial relapse. In fact, patients who received WBRT in conjunction with SRS had a worse overall survival [8].
The European Organization for Research and Treatment of Cancer (EORTC) trial 2295226001 randomized patients with 1-3 metastases to WBRT or observation following SRS or surgical resection of brain metastases. The primary end point for this study was the time to a WHO performance status deterioration of $>2$. Quality of life $(\mathrm{QoL})$ was measured with validated instruments (EORTC QLQ-c30 and EORTC QLQ-BN20). A total of 359 patients were enrolled, with 199 initially treated with SRS and 160 with surgery. Among those patients, 180 were randomized to WBRT and 179 to observation. The primary end point did not significantly differ between the two arms [9]. Intracranial failures were more common in patients observed following focal brain tumor treatment, but overall survival was not jeopardized by withholding WBRT. There were more deaths related to neurologic progression in the group that did not have WBRT, but at least some component of this is from patients who were treated with surgery alone, whose local recurrence rate at 2 years was 59\%, nearly double that for patients treated with SRS alone [9]. The first-year QoL data from this trial were reported separately, and it was observed that patients not treated with WBRT had a better reported QoL. They noted a beneficial score difference of about 10 marks in the domains of global health status, physical functioning, cognitive functioning and fatigue [12]. Therefore, SRS alone results in not only better neurocognitive function but also QoL.

At the most recent American Society of Clinical Oncology (ASCO) meeting in June 2015, Brown et al. presented the results of NCCTG N0574. This Phase III randomized trial recognized that prior investigations had failed to show an impact on survival of WBRT, and used a battery of validated tests to evaluate neurocognitive functioning in patients with $1-3$ brain metastases treated with SRS with or without WBRT to determine which had a worse cognitive impact: WBRT or recurrence of brain metastases. The authors reported a significant decline in neurocognitive performance on the HVLT-R (total and delayed components) and on Controlled Oral Word Association (COWA) testing following WBRT at the primary end point of 3 months that persist at 6 months and also perhaps at a year [10]. These data have, however, only been reported in abstract form. Nevertheless, this is a positive study favoring SRS alone and confirms the results from the Chang et al. MDACC study. Importantly, although intracranial relapse rates were greater 
with SRS alone, this did not adversely impact neurocognitive functioning, and no survival differences were observed [10]. These results, together with those from Chang et al., suggest that WBRT impacts neurocognition independent of recurrent disease $[8,10]$. The salvage therapy rates were high in this population, highlighting the importance of vigilant follow-up and management of new brain metastases that may develop.

An initial attempt to perform a meta-analysis on patients enrolled in brain metastases clinical trials tried to address the issue of survival, but was limited by the way in which the original data were reported [13]. In 2015, Sahgal et al. reported an individual patient data meta-analysis of the MDACC, EORTC and Japanese trials (the results of the NCCTG trial had yet to be reported). Overall, when all patients were analyzed, no survival benefit to WBRT was observed despite lower rates of local and distant brain failure [14]. However, when subgroups were analyzed, an age effect was observed such that patients age 50 years or younger were found to have a survival advantage when treated with SRS alone [14]. A difference was also observed in distant brain failure in this cohort compared with older patients, with no positive impact to adjuvant WBRT with respect to reducing distant failure rates. Therefore, the authors postulated that if exposed to the harms of WBRT without realizing any benefits (with respect to distant brain control), survival can be adversely impacted. This also may explain why in the older patients, there was no survival difference (those $>50$ years) as the expected benefit with respect to distant brain control with adjuvant WBRT was observed [14]. This plausible hypothesis remains to be validated.

Therefore, as outlined in the data above, based on level 1 evidence from four randomized Phase III trials, and an individual patient data meta-analysis, SRS alone should be regarded as a standard of care option for patients with $\leq 4$ metastases. This option is increasingly important in modern oncologic medicine where patients are living to experience the late toxicities of brain irradiation and improvements in systemic control necessitate more durable management of the brain. Furthermore, novel therapeutics with targeted therapies and immunotherapy that penetrate the CNS appear to reduce the development of new brain metastases. Our professional societies have also acknowledged the change in paradigm and SRS alone for patients with limited metastases has been supported by American Society for Radiation Oncology (ASTRO), National Comprehensive Cancer Network (NCCN), German Society of Radiation Oncology (DEGRO) and other guidelines [15-17]. In fact, current trials now focus on SRS alone for $>4$ brain metastases in an attempt to spare more patients from WBRT. Ultimately, the historical approach of brain metastases management based strictly on a number of lesions is overly simplistic. We have evolved from dogmatic teachings and recognize that it is not necessary to treat an entire organ for just a few metastases.

\section{Financial \& competing interests disclosure}

KJ Redmond, A Sahgal and SS Lo are members of an oligometastasis research consortium funded through a grant from Elekta AB. SS Lo has received an honorarium for past educational seminars from Varian Medical Systems. A Sahgal has research grants from Elekta $A B$ and received honorarium for past educational seminars from Elekta $A B$, Medtronic and Varian Medical Systems. The authors have no other relevant affiliations or financial involvement with any organization or entity with a financial interest in or financial conflict with the subject matter or materials discussed in the manuscript apart from those disclosed.

No writing assistance was utilized in the production of this manuscript.

\section{References}

1 Nieder C, Spanne O, Mehta MP, Grosu AL, Geinitz H. Presentation, patterns of care, and survival in patients with brain metastases: what has changed in the last 20 years? Cancer 117, 2505-2512 (2011).

2 Sahgal A, Larson D, Knisely J. Stereotactic radiosurgery alone for brain metastases. Lancet Oncol. 16, 249-250 (2015).

3 Patchell RA, Tibbs PA, Walsh JW et al. A randomized trial of surgery in the treatment of single metastases to the brain. N. Engl. J. Med. 322, 494-500 (1990).
4 Vecht CJ, Haaxma-Reiche H, Noordijk EM et al. Treatment of single brain metastasis: radiotherapy alone or combined with neurosurgery? Ann. Neurol. 33, 583-590 (1993).

5 Andrews DW, Scott CB, Sperduto PW et al. Whole brain radiation therapy with or without stereotactic radiosurgery boost for patients with one to three brain metastases: Phase III results of the RTOG 9508 randomised trial. Lancet 363, 1665-1672 (2004).

6 Patchell RA, Tibbs PA, Regine WF et al. Postoperative radiotherapy in the treatment of single metastases to the brain: a randomized trial. JAMA 280, 1485-1489 (1998).

7 Aoyama H, Shirato H, Tago M et al. Stereotactic radiosurgery plus whole-brain radiation therapy vs stereotactic radiosurgery alone for treatment of brain metastases: a randomized controlled trial. JAMA 295(21), 2483-2491 (2006).

8 Chang EL, Wefel JS, Hess KR et al. Neurocognition in patients with brain metastases treated with radiosurgery or radiosurgery plus whole-brain irradiation: a randomised controlled trial. Lancet Oncol. 10, 1037-1044 (2009). 


\section{EDITORIAL Lo, Redmond, Chang, Foote, Knisely \& Sahgal}

9 Kocher M, Soffietti R, Abacioglu U et al. Adjuvant whole-brain radiotherapy versus observation after radiosurgery or surgical resection of one to three cerebral metastases: results of the EORTC 22952-26001 study. J. Clin. Oncol. 29, 134-141 (2011).

10 Brown PD, Asher AL, Ballman KV et al. NCCTG N0574 (Alliance): a Phase III randomized trial of whole brain radiation therapy (WBRT) in addition to radiosurgery (SRS) in patients with 1 to 3 brain metastases. J. Clin. Oncol. 33(Suppl.), Abstract LBA4 (2015).

11 Aoyama H, Tago M, Kato N et al. Neurocognitive function of patients with brain metastasis who received either whole brain radiotherapy plus stereotactic radiosurgery or radiosurgery alone. Int. J. Radiat. Oncol. Biol. Phys. 68, 1388-1395 (2007).
12 Soffietti R, Kocher M, Abacioglu UM et al. A European Organisation for Research and Treatment of Cancer Phase III trial of adjuvant whole-brain radiotherapy versus observation in patients with one to three brain metastases from solid tumors after surgical resection or radiosurgery: quality-of-life results. J. Clin. Oncol. 31, 65-72 (2013).

13 Tsao M, Xu W, Sahgal A. A meta-analysis evaluating stereotactic radiosurgery, whole-brain radiotherapy, or both for patients presenting with a limited number of brain metastases. Cancer 118, 2486-2493 (2012).

14 Sahgal A, Aoyama H, Kocher M et al. Phase 3 trials of stereotactic radiosurgery with or without whole-brain radiation therapy for 1 to 4 brain metastases: individual patient data meta-analysis. Int. J. Radiat. Oncol. Biol. Phys. 91, 710-717 (2015).
15 National Comprehensive Cancer Network Central Nerous System Cancers. NCCN Clinical Practice Guidelines in Oncology (2013).

16 Tsao MN, Rades D, Wirth A et al. Radiotherapeutic and surgical management for newly diagnosed brain metastasis(es): an American Society for Radiation Oncology evidence-based guideline. Pract. Radiat. Oncol. 2, 210-225 (2012).

17 Kocher M, Wittig A, Piroth MD et al. Stereotactic radiosurgery for treatment of brain metastases. A report of the DEGRO Working Group on Stereotactic Radiotherapy. Strahlentherapie und Onkologie: Organ der Deutschen Rontgengesellschaft [et al.]. 190, 521-532 (2014). 